\title{
Effect of age on dual-task performance in children and adults
}

\author{
Mike Anderson • Romola S. Bucks • Donna M. Bayliss • \\ Sergio Della Sala
}

Published online: 3 May 2011

(C) Psychonomic Society, Inc. 2011

\begin{abstract}
Age effects on dual-task costs were examined in healthy adults (Exp. 1) and in typically developing children (Exp. 2). In both experiments, individual differences in performance on the single-task components were titrated so that any age differences in dual-task costs could not be attributed to differences in single-task performance. Dualtask costs were found, but there were no age-related differences in these costs in older relative to younger adults, in 7-year-old relative to 9-year-old children, or across all four age groups. The results from these experiments suggest that previously reported age differences in dual-task costs, in both healthy ageing and child development, may be due to a failure to adequately equate singletask difficulty.
\end{abstract}

\section{Keywords Dual-task cost · Aging · Development}

Dual tasking is the ability to coordinate performance on two tasks carried out simultaneously. One of the ongoing debates within the memory and attention literature is whether or not there is a cost associated with performing more than one task at a time, with the evidence suggesting that there is a small but detectable dual-task cost, which varies according to the type of single tasks chosen

\footnotetext{
M. Anderson $(\bowtie) \cdot$ R. S. Bucks $\cdot$ D. M. Bayliss

Neurocognitive Development Unit, School of Psychology, University of Western Australia,

35 Stirling Highway, Crawley,

Perth, WA 6009, Australia

e-mail: mike@psy.uwa.edu.au

\section{S. Della Sala}

Human Cognitive Neuroscience and Centre for Cognitive Ageing and Cognitive Epidemiology, University of Edinburgh, Edinburgh, UK
}

(Verhaeghen, Steitz, Sliwinski, \& Cerella, 2003). A second important debate revolves around whether dual-task coordination is affected by age and by intelligence or whether it is largely independent of individual differences in cognitive functioning - with the exception that it might be affected by some specific brain pathologies, particularly dementia (Baddeley, Baddeley, Bucks, \& Wilcock, 2001; Baddeley, Bressi, Della Sala, Logie, \& Spinnler, 1991; Della Sala \& Logie, 2001; MacPherson, Della Sala, Logie, \& Wilcock, 2007; Sebastian, Menor, \& Elosua, 2006). That is, at issue is whether there is a differential dual-task coordination cost for healthy individuals of different ages or with different levels of intelligence.

Studies of dual tasking have typically focused on the investigation of the psychological refractory period (e.g., Levy, Pashler, \& Boer, 2006) or the ability to switch between two tasks (e.g., Monsell, 2003). The present study concentrates instead on dual-task competition for general processing resources, or more specifically, the competition that arises when two tasks are performed simultaneously but do not call for the same basic cognitive functions to be carried out, such as performing a digit span task while tracing a moving target on a screen (e.g., Baddeley \& Della Sala, 1996; Baddeley, Logie, Bressi, Della Sala, \& Spinnler, 1986; Della Sala, Baddeley, Papagno, \& Spinnler, 1995; Logie, Cocchini, Della Sala, \& Baddeley, 2004). Several independent studies have shown that young, healthy individuals can perform these kinds of dual tasks with little or no dual-task cost-that is, the decrement in performance on either task relative to single-task performance is minimal (e.g., Baddeley et al., 2001; Cocchini, Logie, Della Sala, \& MacPherson, 2002; Della Sala, Foley, Beschin, Allerhand, \& Logie, 2010; Duff \& Logie, 2001; Logie et al., 2004; MacPherson et al., 2007). In contrast, individuals even in the early stages of Alzheimer's disease 
appear to show a specific impairment in dual-task performance that cannot be explained in terms of their single-task performance or the effects of overall cognitive demand on a damaged brain (Baddeley et al., 2001; Baddeley et al., 1986; Collette, Van der Linden, Bechet, \& Salmon, 1999; Kaschel, Logie, Kazén, \& Della Sala, 2009; Logie et al., 2004; MacPherson et al., 2007; Sebastian et al., 2006). This has led to the suggestion that there might be a specific mechanism responsible for task coordination: a mechanism that is damaged by Alzheimer's disease.

However, whether or not the task coordination mechanism is affected by age has yet to be resolved. Several authors have suggested that dual-task costs are significantly higher in older than in younger adults, particularly when the demands of the concurrent tasks are high (N. D. Anderson, Craik, \& Naveh-Benjamin, 1998; Craik, 1977; Craik, Govoni, Naveh-Benjamin, \& Anderson, 1996; Craik \& McDowd, 1987; Fernandes \& Moscovitch, 2000; Hartley \& Little, 1999; Linderberger, Marsiske, \& Baltes 2000; McDowd \& Craik, 1988; Naveh-Benjamin, Craik, Guez, \& Kreuger, 2005; Salthouse, Rogan, \& Prill, 1984; Wright, 1981). On the other hand, others have reported no differential age-related dual-task costs (Baddeley et al., 2001; Baddeley et al., 1986; Somberg \& Salthouse, 1982), even when task difficulty was manipulated (Gick, Craik, \& Morris, 1988; Logie et al., 2004; Morris, Gick, \& Craik, 1988).

Two explanations have been put forward to account for this lack of consensus as to whether age affects dual-task costs:

(1) Some studies have equated for single-task difficulty while others have not. Equating removes the problems of differential performance on single tasks, so that any difference can be attributed to a real difference in the cognitive cost of co-coordinating the two tasks- that is, a differential dual-task cost;

(2) Age-related differential dual-task costs have been found when reaction time (RT) is the performance measure for one of the tasks (for a recent discussion, see Logie, Della Sala, MacPherson, \& Cooper, 2007). Thus, in experiments showing a differential dual-task cost between older and younger groups, individual differences in single-task RT performance have not been titrated.

Salthouse et al. (1984) posited that differences in singletask performance would become paramount under dual-task conditions. Hence, to properly investigate a possible dualtask coordination impairment, it is necessary to equate performance on both individual task components. By titrating tasks for individual ability levels, any changes in performance between single- and dual-task conditions for either group could be attributed specifically to the demands of performing two tasks concurrently, rather than being considered to reflect differences between groups in their ability to perform the two component tasks. Consistent with this view, age-related dual-task effects are not observed (or are minimal) when the different age groups have been equated for single-task performance on both tasks (Baddeley et al., 1991; Baddeley et al., 1986; Belleville, Rouleau, \& Caza, 1998; Hartley \& Little, 1999; Logie et al., 2004; Parkinson, Lindholm, \& Urell, 1980; Salthouse, Fristoe, Lineweaver, \& Coon, 1995; Somberg \& Salthouse, 1982).

By contrast, and of particular relevance to the present study, many studies that have shown a differential age effect on dual-task cost have used RT paradigms as one of the two component tasks. For example, Macht and Buschke (1983) found no significant age-related difference in dual-task costs in memory performance, but did find an age-related decrement on concurrent RT (see also Naveh-Benjamin et al., 2005). Similarly, Logie et al. (2007) observed that older adults do show differential dual-task costs, but only when one of the tasks involves a speeded response. Clearly, there is a need to equate task difficulties for speeded task performance to determine whether there is something special about speed as a predictor of dual-task cost. However, it is extremely difficult to equate RT performance across different age groups. The extent to which this can be done is necessarily post hoc (subsamples of individuals with roughly similar RTs can be selected from within the two age groups - see Logie et al., 2007) and beyond experimental control (titration). It is, therefore, possible that age affects dual-task performance on tasks that involve RT, but the question is left open whether age affects dual-task performance when single-task demands (including those on speeded tasks) are properly adjusted to the abilities of individual participants. For this reason, we chose an inspection time (IT) task as one of our component tasks. IT is a commonly used measure of the speed of information processing (Nettelbeck, 1987), but because it involves varying the exposure duration of a stimulus to obtain a predetermined level of accuracy, it is by definition a titrated measure of speed. Consequently, by using IT as our titrated speed measure, we were able to determine whether there is something special in the relationship between speed of processing and differential dual-task costs, or whether speeded tasks have shown this differential cost because of the inability, heretofore, to titrate individual task difficulty to adjust for individual differences in speed of processing.

While much of the present interest in dual-task costs has focused on the effect of cognitive ageing, a long line of developmental literature has also examined changes in dual-task performance in children. Much of this research has focused on whether changes in cognitive capacity might be invoked to explain changes in the ability of children to perform cognitive tasks of increasing complexity (Halford, Maybery, \& Bain, 1986; Pascual-Leone, 1970). The consensus from such research has been that it appears that 
older children have more spare cognitive capacity (as assessed by the influence of a primary on a secondary task) and in dualtask procedures show reduced dual-task costs (Guttentag, 1989). While some of this research has attempted to control for the difficulty of single-task performance statistically, usually by partialling out developmental differences in single-task performance in some manner (see, e.g., the easy-to-hard paradigm of Hunt \& Lansman, 1982), there is a suggestion that proper control of single-task difficulty can only be achieved using experimental rather than statistical control methods. Irwin-Chase and Burns (2000) used titration experimentally to equate single-task difficulty in a dual-task procedure. They equated for task difficulty by varying the exposure duration of two perceptual detection tasks so that these tasks were equally difficult for children of different ages (8- and 11-year-olds). When this was done, no age-related difference in dual-task cost was found.

The aim of the present study was to investigate the effect of age on dual-task performance when both tasks were titrated for individual differences. As noted, differential age-related dual-task costs have been found only in latency or speed measures, not in accuracy (Verhaeghen et al. 2003), and previous studies using speed tasks have used traditional RT measures that, by definition, cannot be titrated. One of the tasks that we use, IT, is a measure of the speed of information processing. This is important because, according to some, an alternative explanation to the 'coordination hypothesis' for the specific dual-task cost in patients with brain pathologies is that the dual-task cost itself depends on differences in speed of processing. However, IT provides a means of titrating for task difficulty in a way that would be impossible with RT tasks. Thus, to our knowledge, this is the first study of age differences in dual-task performance to use a speed task and a digit span task for which we can be confident that equivalent performance is obtained on both tasks when they are completed alone. This allowed us to examine whether age differences in dual-task costs would be evident when both tasks were equated. In Experiment 1, we compared young and older participants, and in Experiment 2 we compared the performance of children of different ages. Children are of particular interest because of the well-known difficulty that young children have in carrying out multiple tasks, which is also the case with Alzheimer's disease. This opens the intriguing possibility that we may detect the maturation of this hypothetical mechanism in the development of children, particularly in the case of children undergoing substantial changes in frontal functioning. Hence, for our study we chose children between the ages of 7 and 9 years old. Thus, this study is novel in its comparison of dual-task costs at the two ends of the lifespan. Given the hypothesis that dual-task coordination evokes a specific coordination mechanism that is unaffected by normal ageing, in Experiment 1 we predicted that when we titrated for task difficulty, there would be a small dual-task cost (around 5\%-10\%, according to the previous literature) but no age-related difference in cost. However, the consensus of the literature from child development led to the prediction that, in contrast to the older adults in Experiment 1, we would find an age effect on dual-task costs in Experiment 2.

\section{Experiment 1}

Method

\section{Participants}

Based on our previous work (Logie et al., 2007) which reported a significant differential age-related dual-task cost, $d=0.62$, a sample of 33 participants per age group would be needed to detect a significant differential dual-task cost with power of .80 and alpha of .05 and a one-sample test. Given that the IT task used in the present study was novel, we sought to recruit a further $25 \%$ of participants. A total of 42 younger participants (33 female) between 17 and 27 years of age $(M=20.8$ years, $S D=2.5)$ and 45 older participants (30 female) between 50 and 81 years of age $(M=61.6, S D=8.32)$ took part in this experiment. The two groups did not differ in terms of their years of education (younger, $M=14.94$ years, $S D=2.42$, range $=10-20$; older, $M=14.65$ years, $S D=5.53$, range $=6-38)$. The younger group was recruited from a pool of undergraduate students and received course credit for their participation, and the older participants were recruited through the West Australian Participant Pool, a panel of community volunteers aged 50+ (Director, RSB), and received an honorarium of $\$ 10$ to cover expenses.

Background assessment All participants were given a number of assessments. They included the Montreal Cognitive Assessment (MoCA; Nasreddine et al., 2005), a cognitive screening test for early signs of dementia; the Australian version of the National Adult Reading Test (AusNART; Hennessy \& Mackenzie, 1995), a method of estimating premorbid, crystallised, verbal IQ; the Letter Number Sequencing subtest of the Wechsler Adult Intelligence Scale (WAIS-III-R; Wechsler, 1997), a measure of working memory; and the Cattell Culture Fair measure of fluid intelligence (Scale 2, Form A; Cattell \& Cattell, 1960).

\section{Tasks and procedure}

Each participant completed a procedure that estimated their inspection time and digit span, followed by single and 
concurrent (dual-task) performance of an IT and a digit recall task.

Digit span estimate The estimate of digit span involved a computerised auditory presentation (in a female voice) of lists of numbers at a rate of one per second. Participants were presented with a list of numbers and required to verbally reproduce the sequence in the correct order. An administrator then input the recalled list into the computer using the keyboard. The procedure commenced with three lists of two digits. If two of these lists were correctly recalled, the digit sequence was increased by one digit. Testing was discontinued when the participant failed to correctly recall two out of the three digit lists at a given length. A digit span estimate was calculated as the maximum list length for which participants were able to correctly recall two out of the three digit sequences. This estimate was then used in the digit recall tasks.

Digit recall (single and dual task) In the single-task version of digit recall, participants were required to recall a computer-presented auditory list of digits presented at a rate of one per second. Following the auditory presentation of each trial, the participant attempted to correctly recall the digits presented (recalling all of the items and in the correct order). The length of the list was the span estimated from the digit span estimation procedure. The number of trials was dependent on the span estimate (those with a span $<4$ had 12 trials; those with a 4 had 10 trials; those with a 5 had 8 trials; those with $>5$ had 6 trials). The variation in the number of trials for the different list lengths was required in order to keep the overall duration of the single task to approximately $2 \mathrm{~min}$ for each participant. An administrator manually recorded the responses for each trial for later scoring. Performance was scored as the percentage of correctly recalled lists. The dual-task version of digit recall was identical to the single-task version, except that it was administered concurrently with IT.

Inspection time estimate The estimate of IT used a computer-based task that is simple to understand and has been used extensively with a variety of participant populations, including typically developing children (M. Anderson, 1988), children with autism (M. Anderson, O'Connor, \& Hermelin, 1998; Scheuffgen, Happé, Anderson, \& Frith, 2000), groups with general mental retardation (M. Anderson \& Miller, 1998; Moore, Hobson, \& Anderson, 1995), individuals with schizophrenia (Badcock, Williams, Anderson, \& Jablensky, 2004), and children at risk of developmental coordination disorder (Piek et al., 2004). The IT task takes the form of a computer game in which "aliens" of different kinds must be discriminated. The IT stimuli consist of alien characters with antennae of the same or of different lengths (see M. Anderson, 1988), subtending three degrees of visual angle in height and two degrees in width, at a viewing distance of $30 \mathrm{~cm}$. Participants were instructed to detect whether the presented alien had the sameor different-length antennae. The stimuli were presented on a personal computer utilizing the Psychophysics Toolbox, Version 2.54 (Brainard, 1997; Pelli, 1997) with MATLAB 7.2 , to drive the monitor. The monitor was a ViewSonic Model E70 with a $100-\mathrm{Hz}$ refresh rate $(10 \mathrm{~ms} /$ frame $)$. Exposure duration was controlled using a dynamic backward mask (Knibb, 1992). This consisted of two pattern masks; the first was presented at the end of the stimulus exposure duration, followed $30 \mathrm{~ms}$ later by a different pattern mask (to create the dynamic masking effect). Each mask remained on the screen until the beginning of the next trial. Each trial was initiated by the participant and commenced with a blank interval during which a black background was presented $(200 \mathrm{~ms})$, followed by a fixation cross indicating the central location of the to-be-presented target $(200 \mathrm{~ms})$, then the target stimuli (their exposure determined according to a parameter estimation by sequential testing [PEST] procedure, described below), followed by a dynamic backward mask. Correct responses were followed by a high-pitched tone, and incorrect responses were followed by a lower tone.

The exposure duration of the stimuli was varied in three independent staircases using a PEST procedure. PEST is an adaptive procedure that uses an algorithm based on the proportion of correct responses at any particular exposure duration to determine whether the desired level of accuracy will be approached by increasing or decreasing the exposure duration of the stimulus. In this way, the algorithm "hones in" on a preset accuracy threshold. The three independent adaptive staircases all estimated the $70 \%$ accuracy threshold. The first staircase consisted of the first 25 trials and served as practice. The initial exposure duration was $160 \mathrm{~ms}$. On completion of the practice staircase, two remaining test staircases were interleaved for 50 trials each. The first trial of each of these staircases began with the final exposure duration from the practice staircase. The 100 test trials (50 per staircase) were presented in four equal blocks of 25 trials. Each of these blocks was preceded by three practice trials with a long exposure duration of $160 \mathrm{~ms}$. In total, then, there were 137 IT trials. The mean exposure duration of the final 5 trials for each of the two test staircases was calculated, and the IT estimate was taken to be the mean of these two means. Testing took approximately $5 \mathrm{~min}$.

Inspection time (single and dual task) The single-task version of the IT task consisted of 25 trials with a constant 
stimulus exposure duration that was determined by the IT estimation procedure. In addition, there were an initial 2 practice trials on which the exposure duration was set at the IT estimate plus $50 \mathrm{~ms} .{ }^{1}$ Following a correct response, participants were presented with a 'visual explosion' for feedback. ${ }^{2}$ After the feedback, the next trial began after a 200-ms blank interval, during which the black background was present. If an incorrect response was made, the next trial began after a 400-ms blank interval. The performance measure was percent correct responses. The dual-task version of the IT task was identical to the single-task version, except that it was administered concurrently with digit recall.

Dual tasks Digit recall and IT task performance was assessed under both single- and dual-task conditions. Single-task performance was always assessed prior to dual-task performance, and all of the participants completed the single IT task followed by the single digit recall task. In the dual-task condition, participants were asked to perform the IT task at the same time as they listened to and repeated back the digit sequences they heard. This was done for as long as it took each participant to complete a total of 27 IT trials ( 2 practice and 25 tests trials), which was approximately 2 min for each participant. Given the short duration of each IT trial, there were multiple IT trials presented during each digit recall trial. In turn, because of the variable length of the digit recall sequences, individuals with longer spans had fewer digit recall trials overall. The performance measure was the percentage of correct trials for each task, as it was for the single-task versions. However, as articulated by Logie et al. (2004), age group differences in performance on individual tasks under dual-task conditions may not account for trade-offs in performance between the two tasks, or combined changes in performance across the two tasks. Thus, the critical measure for assessing age differences in dual-task costs was a combined index of the percentage change in accuracy when performing the task singly or in combination, for both the digit span and IT tasks. The dualtask cost was calculated for each task in turn:

\section{Percentage change \\ $=($ single-task performance - dual-task performance $\times 100)$ /( single-task performance $)$}

Then, a combined dual-task cost was calculated by averaging the costs for both tasks:

Combined

$=($ percentage change in IT + percentage change in digit recall $) / 2$.

\footnotetext{
${ }^{1}$ Because the exposure duration is limited by the screen refresh rate, these durations were rounded to the nearest $10 \mathrm{~ms}$.

${ }^{2}$ Visual feedback was chosen in order to avoid conflict with the voiced digits in the digit recall task.
}

\section{Results}

\section{Data screening}

Prior to analysis, the data from 6 older participants were excluded due to failure to complete the task because of computer problems, and from 1 additional older participant due to their failure to understand the requirements of the IT task. All remaining participants performed above the cut-off criterion for early signs of dementia on the MoCA of 23/30 (Lee et al., 2008). This left a sample of 42 younger and 38 older adults for the full dual-task procedure.

\section{Intelligence test scores}

Descriptive statistics for the AusNART and the Cattell Culture Fair Intelligence Test (CCF) are displayed in Table 1. Two participants (both older) did not complete the CCF due to time constraints, and predicted IQ from the AusNART could not be calculated for a further 2 participants because their years of education were unknown ( 1 older and 1 younger). The AusNART-predicted WAIS-III Verbal IQ was calculated according to the formula proposed by Sullivan, Senior, and Hennessy (2000). Independentsamples $t$ tests were used to compare younger and older adults on the AusNART and CCF. Older adults had significantly higher predicted verbal IQs than did the younger adults, $t(76)=3.73, p<.001$. However, as expected, the younger adults had significantly higher fluid intelligence scores on the CCF, $t(76)=5.90, p<.001$.

\section{Inspection time and digit span estimates}

The estimates of IT and digit span used to titrate single-task difficulty are presented in Table 2 . The older group had significantly longer ITs than the younger group, $t(78)=7.83$, $p<.001$, but there was no difference between the groups on digit span, $t(78)=1.58$, n.s.

Table 1 Means and standard deviations of scores on the Montreal Cognitive Assessment (MoCA), AusNART predicted WAIS III Verbal IQ, and Cattell Culture Fair Intelligence Test (CCF) for younger and older adults

\begin{tabular}{|c|c|c|c|c|}
\hline & \multicolumn{2}{|c|}{ Younger Adults } & \multicolumn{2}{|c|}{ Older Adults } \\
\hline & \multicolumn{2}{|l|}{$(n=42)$} & \multicolumn{2}{|c|}{$(n=38)$} \\
\hline & $\mathrm{M}$ & SD & $\mathrm{M}$ & $\mathrm{SD}$ \\
\hline MoCA (max. 30) & 28.00 & 1.41 & 27.76 & 1.42 \\
\hline AusNART predicted IQ & 105.37 & 6.84 & 111.16 & 6.86 \\
\hline CCF (max. 46) & 36.69 & 4.05 & 31.25 & 4.07 \\
\hline
\end{tabular}


Table 2 Means and standard deviations of digit span (number of letters) and inspection time (IT, in milliseconds) estimates for younger and older adults

\begin{tabular}{|c|c|c|c|c|}
\hline & \multirow{2}{*}{\multicolumn{2}{|c|}{$\frac{\text { Younger Adults }}{(n=42)}$}} & \multirow{2}{*}{\multicolumn{2}{|c|}{$\begin{array}{l}\text { Older Adults } \\
(n=38)\end{array}$}} \\
\hline & & & & \\
\hline & $\mathrm{M}$ & $\mathrm{SD}$ & M & $\mathrm{SD}$ \\
\hline IT estimates & 39.43 & 12.12 & 68.08 & 20.01 \\
\hline Digit span estimates & 6.00 & 0.99 & 5.63 & 1.10 \\
\hline
\end{tabular}

Table 3 shows percent correct IT performance in the single- and dual-task conditions. There was a main effect of condition, indicating a significant decrease in accuracy in the dual-task as compared with the single-task condition, $F(1,78)=5.07, p<.05, \eta_{\mathrm{p}}^{2}=.06$. However, there was no significant group difference, $F(1,78)=2.0$, n.s., $\eta_{\mathrm{p}}^{2}=.03$, nor was there a condition by group interaction, $F(1,78)=$ 1.65 , n.s., $\eta_{\mathrm{p}}^{2}=.02$. Table 3 also shows percent correct performance on digit recall in the single- and dual-task conditions. There was a main effect of condition indicating a significant decrease in accuracy in the dual-task compared with the single-task condition, $F(1,78)=17.68, p<.001$, $\eta_{\mathrm{p}}^{2}=.19$. However, again there was no significant difference between the groups, $F(1,78)<1$, nor was there a condition by group interaction, $F(1,78)<1$.

\section{Dual-task cost}

Two extreme outliers (i.e., $>3 S D$ s from the mean index of combined dual-task cost) were identified, both in the younger group (combined costs of $-128 \%$ and $-175 \%$, respectively, indicating much better performance in the dual- than in the single-task condition), and these were removed from the dual-task cost analysis. There were no outliers in the older group. A $t$ test comparing the combined dual-task costs for younger $(M=10.10, S D=24.11)$ and older $(M=11.41, S D=24.82)$ participants revealed no significant difference between the groups, $t(76)<1, d=0.05^{3}$ Nonetheless, a subsequent one-sample $t$ test for the age groups combined revealed a significant combined dualtask cost greater than zero, $t(77)=3.90, p<.001,95 \%$ $\mathrm{CI}=5.25-16.22$.

Given that titration is designed to control for preexisting differences in single-task performance, it may be a concern to some that there were no age differences in digit span single-task performance prior to titration. As a


cost outliers made no difference to the effects reported.
}

Table 3 Percent correct for single- and dual-task inspection time (IT) and single- and dual-task digit recall for younger and older adults

\begin{tabular}{llllll}
\hline & \multicolumn{2}{l}{ Younger Adults } & & \multicolumn{2}{l}{ Older Adults } \\
\cline { 2 - 3 } \cline { 5 - 6 } & & & \multicolumn{2}{l}{$(n=38)$} & \\
\cline { 2 - 3 } \cline { 5 - 6 } & $\mathrm{M}$ & $\mathrm{SD}$ & & $\mathrm{M}$ & $\mathrm{SD}$ \\
\hline IT single-task & 63.43 & 15.47 & & 61.26 & 16.69 \\
IT dual-task & 61.90 & 14.03 & & 55.68 & 13.65 \\
Digit recall single-task & 68.45 & 27.00 & & 69.43 & 23.07 \\
Digit recall dual-task & 57.35 & 29.41 & & 56.27 & 28.12 \\
\hline
\end{tabular}

final test, under conditions that were likely to maximise the chances of finding a differential dual-task cost, two groups of younger and older adults were selected to differ on untitrated digit recall performance, and their combined dual-task cost was then reanalysed. For the young adult group, all participants who scored at or above the 25th percentile on the untitrated estimate of digit span were selected $(N=40)$, and for the older adult group all participants who scored at or below the 75th percentile were selected $(N=30)$. This had the desired effect, with a $t$ test comparing the untitrated digit estimates showing a significant difference between the young $(M=$ $6.00, S D=1.01)$ and the older $(M=5.23, S D=0.86)$ adult groups, $t(68)=3.34, p<.01$. However, there were still no differences in combined dual-task costs between the younger and older participants, once performance on both single tasks had been equated using titration, $t(68)<1$.

\section{Discussion}

Previous studies of dual-task performance have demonstrated minimal dual-task costs when healthy individuals perform two tasks simultaneously that had previously been titrated for individual performance. These findings are thought to reflect the operation of a specific dual-task coordination mechanism within a multiple-component working memory system, rather than reflecting general cognitive demands on a central processing resource (Logie et al., 2004). In contrast, even when both single tasks have been equated for individual performance, patients in the early stages of Alzheimer's disease show dramatically larger dual-task costs (Baddeley et al., 1991; Della Sala, Cocchini, Logie, Allerhand, \& MacPherson 2010; MacPherson et al., 2007). This overall picture that has emerged from the previous literature does not hold when one of the two individual tasks is speeded. Under these experimental conditions, older people have proven to be more sensitive to time pressure in responding under dual-task conditions (Logie et al., 2007). However, in the Logie et al. (2007) 
study, RTs could only be equated across age groups a posteriori. Moreover, older adults produced significantly slower RTs than did younger adults in all conditions, and especially when they performed the dual task, leaving open the question of whether or not ageing affects dual tasking when one or the other of the component tasks cannot be fully equated for individual performance.

The methods used in the present study allowed us to investigate this issue directly by offering the possibility of titrating a speeded task. That our older participants needed on average $70 \%$ longer ITs to achieve accuracy equivalent to that of the younger adults underscores the need for titration. Following experimental manipulation to ensure equivalent performance in both single tasks, no differential dual-task cost due to ageing emerged, and this was true despite significant age group differences in fluid intelligence, in the favour of the younger adults. ${ }^{4}$ Moreover, the present results allow us to reconcile the different findings in the ageing literature by claiming that dual-task decrements depend on the particular tasks chosen, particularly on whether the concurrent tasks have been equated for individual ability levels of single-task performance. The finding that any differential dual-task cost associated with ageing is not evident if single-task difficulty is equated opens the possibility that this might happen in child development too. Experiment 2 was designed to investigate this possibility.

\section{Experiment 2}

This experiment was designed to see whether the effects reported in Experiment 1 could be replicated for children. If so, we might expect to find age differences on IT and digit recall (such differences have been reported before) but no differences in dual-task costs. On the other hand, there are a number of reasons to hypothesise that in a child sample we would find age-related effects. First, the older cognitive capacity literature that has explored dual tasking in children has typically reported that older children have lower dual-task costs than do younger children. However,

\footnotetext{
${ }^{4}$ A reviewer expressed concern that the interaction between age group (younger, older) and task (single, dual) in the adult participants on IT accuracy was not significant, and proposed that we failed to find an effect because of power. On the contrary, we did have power (.82) to find an effect, but did not do so. More importantly, however, the combined dual-task cost measures (which are both more sensitive to overall dual-task costs across the two tasks and control for individual or group differences in the allocation of attention between the two tasks) not only were not significantly different between the younger and older adults but, to achieve a significant difference, we would have needed 5,478 participants per group.
}

just as in the case of many studies in the ageing literature, those studies have not featured titration for single-task difficulty. Second, the ages of the children in the present experiment were chosen to maximise the chance that they would be on either side of marked development in frontal functioning (Huttenlocher \& Dabholker, 1992; Thatcher, 1991; Thompson-Schill, Ramscar, \& Chrysikou, 2009).

If dual-task coordination is a component of executive functioning, which is, in turn, dependent on frontal development, then in contrast to what we found in Experiment 1, we might expect to see a difference in costs between the groups.

\section{Method}

\section{Participants}

A total of 114 typically developing children (57 girls) 7 and 9 years of age were recruited from 16 Perth suburban primary schools as part of the Project K.I.D.S. (Kids Intellectual Development Study) program. Project K.I.D.S. is an ongoing research program conducted annually since 1995 at the University of Western Australia. It investigates the development of cognitive, social, and emotional functions in children (see M. Anderson, Reid, \& Nelson, 2001, for a more general description). Only children meeting the requirements of having English as a first language and not having previously attended Project K.I.D.S. were recruited. Ten of the children had missing data due to equipment failures. This left 55 participants who were 7-year-olds $(M=7.53, S D=0.26), 21$ girls and 34 boys, and 49 who were 9-year-olds $(M=9.38, S D=0.25), 27$ girls and 22 boys, who participated in the dual-task study.

Background assessment All children were given a number of assessments as part of Project K.I.D.S. The Cattell Culture Fair Test was part of the battery, and the children were given the same version (Scale 2, Form A) used in Experiment 1. This test was administered in groups of four with children seated at individual desks. A full Wechsler Intelligence Scale for Children (WISC-III, Wechsler, 1991) was also administered. As part of this battery, the Forward Digit Span subtest was administered to each child in a oneon-one testing session according to the standard instructions from the WISC-III manual.

\section{Procedure}

Each participant completed a procedure that estimated their IT and digit span, followed by titrated single and concurrent (dual-task) performance of an IT and a digit recall task. Digit recall was administered in the same way as in 
Experiment 1, with the exception that the length of the digit lists was set as the digit span derived from the child's forward digit span on the WISC-III. IT was estimated using a slightly different PEST procedure (one staircase of 100 trials instead of two interleaved staircases of 50 trials) and was again estimated as the mean of the last five exposure durations. Otherwise, the design and procedure were the same as in Experiment 1.

Results

\section{Data screening}

Five children (4 of the 7-year-olds and 1 of the 9-year-olds) were identified as having extreme scores on their estimated ITs (IT $>150 \mathrm{~ms}$ ), and their performance was not analysed further. This left 51 children in the 7-year-old group and 48 in the 9-year-old group.

\section{Intelligence test scores}

As expected, 9-year-olds had significantly higher fluid intelligence $(\mathrm{CCF})$ scores $(M=30.65, S D=5.21)$ than the 7-year-olds $(M=25.41, S D=5.73), t(97)=4.74, p<.001$. However, the 9-year-olds' full-scale IQ $(M=112.87, S D=$ 11.73) was not significantly different to the 7-year-olds' full-scale IQ $(M=112.45, S D=11.16)$.

\section{Inspection time and digit span estimates}

The estimates of IT and digit span used to titrate singletask difficulty are presented in Table 4 . The 9-year-olds had significantly shorter ITs than the 7-year-olds, $t(97)=$ $1.93, p<.05$, one-tailed, but there was no difference between the groups on digit span, $t(97)<1$.

Table 5 shows percent correct performance on IT in the single- and dual-task conditions. There was a main effect of condition, indicating a significant decrease in accuracy in the dual-task compared with the single-task condition, $F(1,97)=11.00, p<.01, \eta_{\mathrm{p}}^{2}=.10$. There was no

Table 4 Means and standard deviations of inspection time (IT, in milliseconds) and digit span (number of letters) estimates for younger and older children

\begin{tabular}{|c|c|c|c|c|}
\hline & \multicolumn{2}{|c|}{ 7-year-olds } & \multicolumn{2}{|c|}{ 9-year-olds } \\
\hline & \multicolumn{2}{|c|}{$(n=51)$} & \multicolumn{2}{|c|}{$(n=48)$} \\
\hline & $\mathrm{M}$ & SD & $\mathrm{M}$ & $\mathrm{SD}$ \\
\hline IT estimates & 81.86 & 32.20 & 70.11 & 28.16 \\
\hline Digit span estimates & 4.37 & 0.85 & 4.54 & 1.05 \\
\hline
\end{tabular}

Table 5 Percent correct for single- and dual-task inspection time (IT) and single- and dual-task digit recall for younger and older children

\begin{tabular}{|c|c|c|c|c|}
\hline & \multicolumn{2}{|c|}{7 -year-olds } & \multicolumn{2}{|c|}{ 9-year-olds } \\
\hline & \multicolumn{2}{|c|}{$(n=51)$} & \multicolumn{2}{|c|}{$(n=48)$} \\
\hline & M & SD & M & SD \\
\hline IT single-task & 70.35 & 17.49 & 68.25 & 19.64 \\
\hline IT dual-task & 63.92 & 16.83 & 63.08 & 16.63 \\
\hline Digit recall single-task & 88.51 & 15.27 & 87.15 & 18.96 \\
\hline Digit recall dual-task & 79.61 & 23.40 & 80.06 & 21.89 \\
\hline
\end{tabular}

significant difference between the groups, $F(1,97)<1$, nor was there a condition by group interaction, $F(1,97)<1$. Table 5 also shows digit recall accuracy in the single- and dual-task conditions. There was a main effect of condition, indicating a significant decrease in accuracy in the dual-task compared with the single-task condition, $F(1,97)=12.97$, $p<.01, \eta_{\mathrm{p}}{ }^{2}=.12$. There was no significant difference between the groups, $F(1,97)<1$, nor was there a condition by group interaction, $F(1,97)<1$.

\section{Dual-task cost}

A $t$ test comparing the combined dual-task costs for 7 year-olds $(M=6.49, S D=23.19)$ and 9-year-olds $(M=$ 3.37, $S D=20.04)$ revealed no significant difference between the groups, $t(97)<1, d=0.14$. As in Experiment 1 , a subsequent one-sample $t$ test for the age groups combined revealed a significant combined dual-task cost greater than zero, $t(98)=2.29, p=.024,95 \% \mathrm{CI}=0.66-9.30$.

Similar to Experiment 1, a subset of 7- and 9-year-olds was selected to differ in terms of untitrated digit span estimates, and their combined dual-task cost was reanalysed. For the 7-year-olds, all participants scoring at or below the 75th percentile were selected $(N=46)$, and for the 9-year-olds, all participants scoring at or above the 25th percentile were selected $(N=41)$. These groups differed in terms of their untitrated digit span estimates, $t(85)=$ $3.57, p<.01$, with higher digit span estimates for the older $(M=4.80)$ relative to the younger $(M=4.20)$ group, but there were no differences between the groups in combined dual-task costs once performance on both single tasks was equated, $t(85)<1$.

\section{Discussion}

Based on an extensive literature that has shown older children are often more proficient at dual tasking than younger children, we predicted that 7-year-olds would show a greater dual-task cost than 9-year-olds. Contrary 
to this prediction, our manipulation of titrating task difficulty resulted in the same finding in children as in our adults in Experiment 1, of no age-related differences in dual-task costs. ${ }^{5}$

The results from this experiment argue that previous research indicating poorer dual tasking in younger than in older children may have resulted not so much from task coordination or task scheduling difficulties, but from fewer general processing resources. Of course, it might be argued that we did not choose a broad enough age span in children to detect differences. There are a number of arguments to the contrary. First, as reviewed in the introduction, many previous studies have shown developmental differences in dual-task coordination across similar age ranges. Second, the actual cost of dual tasking is close to young adult levels in any case. Third, this 2-year age difference in children resulted in about one standard deviation difference in fluid intelligence (as indicated by CCF scores), which is about the same as the difference between our young and older adults, indicating similar levels of group differences.

\section{Combined analysis}

Both of the experiments above reported the same absence of an age difference in the cost of performing two tasks at once, either in children or in adults. We recognise that this is an extremely challenging finding and that some readers may harbour concerns about whether the studies were sufficiently powered to find the critical age-related effects. Although our sample sizes were sufficient, and even large for this field, and we have shown that either the experiments above were sufficiently powered to find the critical effects, or that age-related differences were so small that they would have required unreasonable numbers of participants to uncover, one way to robustly address this issue is to compare the age groups in a cross-experiment analysis. This not only enables us to increase power by increasing participant numbers, but it also has the benefit of increasing the age range across which we can explore age-related differences in dual-task costs. For the sake of completeness, we have done this in two ways. First, we repeated the single- versus dual-task analyses and the combined dualtask analysis across all four age groups (7-year-olds, 9-yearolds, young adults, older adults). Second, as recommended by a reviewer, we repeated these analyses for 7-year-olds

\footnotetext{
${ }^{5}$ A reviewer noted that the average combined dual-task cost appeared to reduce by $50 \%$ between the ages of 7 and 9 (from $6.49 \%$ to $3.37 \%$ ). However, given the magnitude of this effect, with $80 \%$ power and alpha at .05 (two-tailed), we would need 759 children in each age group for an effect of this magnitude to be significant.
}

and young adults only, given that these groups maximally differ in cognitive development. These analyses confirm our earlier findings.

That is, as expected, we found significant differences between the four age groups for IT, $F(3,175)=2.97, p=$ $.033, \eta_{\mathrm{p}}^{2}=.05$, and digit span, $F(3,175)=15.84, p<.001$, $\eta_{\mathrm{p}}^{2}=.21$, and effects of task for IT, $F(1,175)=15.08, p<$ $.001, \eta_{\mathrm{p}}^{2}=.08$, and for digit span, $F(1,175)=31.59, p<$ $.001, \eta_{\mathrm{p}}^{2}=.15$, but no interactions between task and age group (for both IT and digit span, $F<1$ ). That is, there was a significant cost of carrying out the dual task, but the magnitude of this cost did not differ across the 7- and 9year-olds and the younger and older adults. Moreover, there was no significant difference between the four age groups in combined dual-task cost, $F(3,173)=1.09, p=.356$, $\eta_{\mathrm{p}}^{2}=.02$. $^{6}$ Further, with the exception of the age effect for IT, where there were no differences when performance was collapsed across the single- and dual-task conditions, all effects were the same when comparing 7-year-olds with young, healthy adults [age group effects: IT, $F(1,91)=2.36, p=.128, \eta_{\mathrm{p}}^{2}=.03$, and digit span, $F(1,91)=25.11, p<.001, \eta_{\mathrm{p}}^{2}=.22$; task effects: IT, $F(1,91)=5.59, p=.020, \eta_{\mathrm{p}}^{2}=.06$, and digit span, $F(1,91)=$ 14.13, $p<.001, \eta_{\mathrm{p}}^{2}-.13$; interaction effects: IT, $F(1,91)=$ 2.13, $p=.148, \eta_{\mathrm{p}}^{2}-.02$, and digit span, $\left.F<1\right]$. Likewise, there was no significant difference between 7-years-olds and young adults in combined dual-task costs, $t(89)<1, d=0.15{ }^{7}$

\section{General discussion}

Taken together, these two experiments provide convincing evidence that previously reported differences in developmental dual-task costs, whether early or later in life, may arise because of a methodological failure to adequately equate single-task difficulty, rather than a primary deficit in a specific mechanism responsible for dual-task coordination. When single-task difficulty is equated using this novel experimental procedure, we find a significant dual-task cost in both adults and children, the magnitude of which is consistent with that reported elsewhere (around 3\%-10\%). However, we find no evidence of age differences in the

\footnotetext{
$\overline{{ }^{6} \text { The achieved }}$ power for the interaction between age group and task for IT was .80 . To achieve $80 \%$ power for the same interaction for digit span, we would have needed 260 , or $45 \%$ more, participants. For a significant difference across the four age groups for combined dualtask costs, we would have needed 568 participants.

${ }^{7}$ The achieved power for the interaction between age group and task for IT was .87 . To achieve $80 \%$ power for the same interaction for digit span, we would have needed 826 participants. For a significant difference across the 7-year-olds and adults for combined dual-task costs, we would have needed 679 participants per group.
} 
magnitude of those costs: not in children (7- vs. 9-yearolds), not in adults (younger vs. older), and not across the lifespan. It is difficult to think of any other cognitive process that does not change between the ages of 7 and 70 .

We draw three independent conclusions from the data reported here. First, any such specific mechanism responsible for dual-task coordination is likely to be fully mature by age 7 and does not undergo any significant decrement in performance over the age spans of the adult participants in Experiment 1. Second, given that age group differences in fluid intelligence (both in older relative to younger adults and in 7- relative to 9-year-old children) do not appear to lead to differential dual-task coordination costs, this also suggests that such a dual-task coordination mechanism matures, and declines, independently of fluid intelligence. Finally, it would seem likely, based on the published literature showing a dramatic dual-task cost in Alzheimer's disease and our own finding of no age-related differences in dual-task costs in healthy ageing, that this dual-task coordination mechanism is significantly and specifically impaired in Alzheimer's disease.

We pursued this idea in the context of the hypothesis that the development of such a mechanism would be underpinned by the maturation of the prefrontal cortex. Our findings speak against this. First, the youngest children's dual-task performance did not come close to the typical pattern we find with Alzheimer's disease. To our surprise, the younger children showed levels of cost similar to those that we, and others, have found across the lifespan. It is tempting to conclude, as we have done, that if there is a specific mechanism that is damaged by Alzheimer's disease, it does not undergo marked developmental change beyond 7 years of age. Of course, it is possible that different combinations of tasks might produce different demands on the coordination mechanism. However, there is no reason to suppose that the functioning of this mechanism would be task dependent. Indeed, evidence from combinations of other tasks, such as Digit Span and Box Crossing (Della Sala, Foley, et al., 2010), Visual Search and Auditory Detection (Baddeley et al., 2001), and even two memory tasks (Cocchini et al., 2002), has supported this view. This leaves open the possibility that, if we had tested even younger children, we would have found a difference in dual-task costs, but the younger the group, the more difficult it is to use these kinds of procedures.

The other main feature of our two experiments is that one of our tasks was a speeded task. There has been debate in the literature about whether there is something special about speed of information processing and its relationship to differential dual-task costs. Previously, there has been difficulty in titrating for differences in individual speed of processing: Although Barrouillet, Gavens, Vergauwe, Gaillard, and Camos (2009) equated the difficulty of a speeded task across age groups at the group level, we are not aware of any previous study that has achieved individual titration for a speed-of-processing measure. We achieved this using an inspection time task. It is an important finding that, having equated single-task performance, we saw no differential age-related dual-task costs when one of the tasks was a speed measure.

This leads us to make the strong claim that, once single-task differences in speed of information processing have been individually titrated, we have demonstrated that there is nothing special about speed for any explanation of differential dual-task costs, because there is no such effect in our data. However, a couple of caveats should be noted. First, it might be argued that we have used a new task (IT) for which differential deficits in dual-task costs have not been established when there is no titration, and this might weaken our case. ${ }^{8}$ To use IT untitrated would mean presenting all participants with the same exposure duration. In practice it is not possible to do this, because accuracy differs too markedly across the groups we are typically interested in comparing. In the case of Experiment 1, for example, if we chose the middle point between the groups - an exposure duration of $54 \mathrm{~ms}-90 \%$ of the young group would have had estimated ITs equal to or better than this, while only $27 \%$ of our older sample could match this level of performance. This duration would result in a ceiling effect for $90 \%$ of the younger group and a floor effect for $75 \%$ of the older group. This being the case, performance under dual-task conditions could realistically only be significantly worse for the younger group. So, untitrated IT would very likely result in an interaction between age group and task condition, but in the opposite direction to that predicted, and it would be a trivial artefact of this design. Second, and perhaps of more theoretical significance, is the possibility that the kinds of speed measured by IT on the one hand and by RT on the other are different. IT can be regarded as a measure of speed of visual encoding. RT involves other aspects of the speed of processing, principally the speed of response selection and execution, that are not present in an IT task. Indeed, M. Anderson, Nettelbeck, and Barlow (1997) argued that IT and RT can be dissociated during child development on exactly this basis. Increasing response selection demands affects younger children much more than older children, but increasing stimulus encoding demands does not. It might be, then, that RT tasks do involve other processing demands that might, indeed, lead to differential dual-task costs with age. However, even if this is true, our study demonstrates that this is a more specific aspect of speed of processing than has previously been considered.

\footnotetext{
${ }^{8}$ We thank an anonymous reviewer for this observation.
} 
Group differences in performance that are related to task difficulty are ubiquitous in the literature but, in our view, are relatively uninformative. As Baddeley et al. (2001) noted, the critical issue is not whether we find group-task interactions in general, but whether group and task interact once singletask difficulty is titrated, and whether the combined dual-task cost, which takes into account any differences in allocation of attention between tasks, changes with age. Our results suggest that this experimental procedure may be useful for those interested in the effects of ageing, since it allows for disambiguation of potential confounding differences that occur due to general slowing, as opposed to deficits in specific mechanisms. Based on this study, we advocate the methodology as a very useful technique for controlling for general processing differences when investigating hypotheses about specific, age-related mechanisms. The most important use of this technique will be the development of a measure for the early detection of disease in conditions such as Alzheimer's (see Della Sala, Foley, et al., 2010, and Kaschel et al., 2009). The cognitive hallmark of Alzheimer's disease is a deficit in episodic memory; such a deficit, however, also affects healthy ageing and conditions that may be confused with dementia, such as chronic depression in the elderly. A measure that is exempt from age effects could assist in the assessment, early diagnosis, and follow-up of people with dementia.

Author Note This research was supported by a Discovery Project grant from the Australian Research Council to M.A. (DP0665616). We thank Silma Sulaiman, Claire Mein, and Danielle Lowe for their work on this project.

\section{References}

Anderson, M. (1988). Inspection time, information processing and the development of intelligence. British Journal of Developmental Psychology, 6, 43-57.

Anderson, N. D., Craik, F. I. M., \& Naveh-Benjamin, M. (1998). The attentional demands of encoding and retrieval in younger and older adults: 1. Evidence from divided attention costs. Psychology and Aging, 13, 405-423.

Anderson, M., \& Miller, K. L. (1998). Modularity, mental retardation, and speed of processing. Developmental Science, 1, 239-245.

Anderson, M., Nettelbeck, T., \& Barlow, J. (1997). Using reaction time measures of speed of information processing: Speed of response selection increases with age but speed of stimulus categorisation does not. British Journal of Developmental Psychology, 15, 145-157.

Anderson, M., O’Connor, N., \& Hermelin, B. (1998). A specific calculating ability. Intelligence, 26, 383-403.

Anderson, M., Reid, C., \& Nelson, J. (2001). Developmental changes in inspection time: What a difference a year makes. Intelligence, 29, 475-486.

Badcock, J., Williams, R., Anderson, M., \& Jablensky, A. (2004). Speed of processing and individual differences in IQ in schizophrenia: general or specific cognitive deficits. Cognitive Neuropsychiatry, 9, 233-247.

Baddeley, A. D., Baddeley, H. A., Bucks, R. S., \& Wilcock, G. K. (2001). Attentional control in Alzheimer's disease. Brain, 124, $1492-1508$.

Baddeley, A. D., Bressi, S., Della Sala, S., Logie, R., \& Spinnler, H. (1991). The decline of working memory in Alzheimer's disease: a longitudinal study. Brain, 114, 2521-2542.

Baddeley, A. D., \& Della Sala, S. (1996). Working memory and executive control. Philosophical Transactions of the Royal Society B, 29, 1397-1403.

Baddeley, A. D., Logie, R., Bressi, S., Della Sala, S., \& Spinnler, H. (1986). Dementia and working memory. Quarterly Journal of Experimental Psychology, 38A, 603-618.

Barrouillet, P., Gavens, N., Vergauwe, E., Gaillard, V., \& Camos, V. (2009). Working memory span development: a time-based resource-sharing model account. Developmental Psychology, 45, 477-490.

Belleville, S., Rouleau, N., \& Caza, N. (1998). Effect of normal aging on the manipulation of information in working memory. Memory \& Cognition, 26, 572-583.

Brainard, D. H. (1997). The psychophysics toolbox. Spatial Vision, 10, 433-436.

Cattell, R. B., \& Cattell, A. K. S. (1960). Culture fair intelligence tests. Savoy, IL: Institute for Personality and Ability Testing.

Cocchini, G., Logie, R. H., Della Sala, S., \& MacPherson, S. E. (2002). Concurrent performance of two memory tasks: evidence for domain specific working memory systems. Memory \& Cognition, 30, 1086-1095.

Collette, F., Van der Linden, M., Bechet, S., \& Salmon, E. (1999). Phonological loop and central executive functioning in Alzheimer's disease. Neuropsychologia, 37, 905-918.

Craik, F. I. M. (1977). Age differences in human memory. In J. E. Birren \& K. W. Schaie (Eds.), Handbook of the psychology of aging (Vol. 1, pp. 384-420). New York: Van Nostrand Reinhold.

Craik, F. I. M., Govoni, R., Naveh-Benjamin, M., \& Anderson, N. D. (1996). The effects of divided attention on encoding and retrieval processes in human memory. Journal of Experimental Psychology: General, 125, 159-180.

Craik, F. I. M., \& McDowd, J. M. (1987). Age differences in recall and recognition. Journal of Experimental Psychology: Learning, Memory, and Cognition, 13, 474-479.

Della Sala, S., Baddeley, A., Papagno, C., \& Spinnler, H. (1995). Dual-task paradigm: A means to examine the central executive. Annals of the New York Academy of Sciences, 769, 161-171.

Della Sala, S., Cocchini, G., Logie, R. H., Allerhand, M., \& MacPherson, S. E. (2010). Dual task during encoding, maintenance and retrieval in Alzheimer disease and healthy ageing. Journal of Alzheimer's Disease, 19, 503-515.

Della Sala, S., Foley, J. A., Beschin, N., Allerhand, M., \& Logie, R. H. (2010). Assessing dual-task performance using a paper-and-pencil test: Normative data. Archives of Clinical Neuropsychology, 25, 410-419.

Della Sala, S., \& Logie, R. H. (2001). Theoretical and practical implications of dual-task performance in Alzheimer's disease. Brain, 124, 1479-1481.

Duff, S. C., \& Logie, R. H. (2001). Processing and storage in working memory span. Quarterly Journal of Experimental Psychology, $54 A, 31-48$.

Fernandes, M. S., \& Moscovitch, M. (2000). Divided attention and memory: Evidence of substantial interference effects at retrieval and encoding. Journal of Experimental Psychology: General, 129, 155-176.

Gick, M. L., Craik, F. I. M., \& Morris, R. G. (1988). Task complexity and age differences in working memory. Memory \& Cognition, $16,353-361$. 
Guttentag, R. E. (1989). Age-differences in dual-task performanceprocedures, assumptions, and results. Developmental Review, 9, $146-170$

Halford, G. S., Maybery, M. T., \& Bain, J. D. (1986). Capacity limitations in children's reasoning: a dual-task approach. Child Development, 57, 616-627.

Hartley, A. A., \& Little, D. M. (1999). Age-related differences and similarities in dual-task interference. Journal of Experimental Psychology: General, 128, 416-449.

Hennessy, M., \& Mackenzie, B. D. (1995, November). AUSNART: The development of an Australian version of the NART. Paper presented at the 18th Annual Brain Impairment Conference, Hobart, Tasmania.

Hunt, E., \& Lansman, M. (1982). Individual differences in attention. In R. Sternberg (Ed.), Advances in the psychology of human intelligence, vol. 1 (pp. 207-254). Hillsdale, NJ: Erlbaum.

Huttenlocher, P. R., \& Dabholker, A. S. (1992). Regional differences in synaptogenesis in human cerebral cortex. Journal of Comparative Neurology, 387, 167-178.

Irwin-Chase, H., \& Burns, B. (2000). Developmental changes in children's abilities to share and allocate attention in a dual task. Journal of Experimental Child Psychology, 77, 61-85.

Kaschel, R., Logie, R., Kazén, M., \& Della Sala, S. (2009). Alzheimer's disease, but not ageing or depression, affects dualtasking. Journal of Neurology, 256, 1860-1868.

Knibb, K. (1992). A dynamic mask for inspection time. Personality \& Individual Differences, 13, 237-248.

Lee, J. Y., Lee, D. W., Cho, S. J., Na, D. L., Jeon, H. J., Kim, S. K., et al. (2008). Brief screening for mild cognitive impairment in elderly outpatient clinic: Validation of the Korean version of the Montreal Cognitive Assessment. Journal of Geriatric Psychiatry and Neurology, 21, 104-110.

Levy, J., Pashler, H., \& Boer, E. (2006). Central interference in driving: Is there any stopping the psychological refractory period? Psychological Science, 17, 228-235.

Linderberger, U., Marsiske, M., \& Baltes, P. B. (2000). Memorizing while walking: Increase in dual-task costs from young adulthood to old age. Psychology and Aging, 15, 417-436.

Logie, R. H., Cocchini, G., Della Sala, S., \& Baddeley, A. D. (2004). Is there a specific executive capacity for dual task co-ordination? Evidence from Alzheimer's disease. Neuropsychology, 18, 504513.

Logie, R. H., Della Sala, S., MacPherson, S. E., \& Cooper, J. (2007). Dual task demands on encoding and retrieval processes: Evidence from healthy adult ageing. Cortex, 43, 159-169.

Macht, M. L., \& Buschke, H. (1983). Age differences in cognitive effort in recall. Journal of Gerontology, 38, 695-700.

MacPherson, S. E., Della Sala, S., Logie, R. H., \& Wilcock, G. K. (2007). Specific AD impairment in concurrent performance of two memory tasks. Cortex, 43, 858-865.

McDowd, J. M., \& Craik, F. I. M. (1988). Effects of aging and task difficulty on divided attention performance. Journal of Experimental Psychology: Human Perception and Performance, 14, 267-280.

Monsell, S. (2003). Task switching. Trends in Cognitive Sciences, 7, 134-140.

Moore, D., Hobson, R. P., \& Anderson, M. (1995). Direct perception: Evidence for IQ independent processing. Intelligence, 20, 65-86.

Morris, R. G., Gick, M. J., \& Craik, F. I. M. (1988). Processing resources and age differences in working memory. Memory \& Cognition, 16, 362-366.
Nasreddine, Z. S., Phillips, N. A., Bedirian, V., Charbonneau, S., Whitehead, V., Collin, I., et al. (2005). The montreal cognitive assessment, moca: a brief screening tool for mild cognitive impairment. Journal of the American Geriatrics Society, 53, 695699.

Naveh-Benjamin, M., Craik, F. I. M., Guez, J., \& Kreuger, S. (2005). Divided attention in younger and older adults: Effects of strategy and relatedness on memory performance and secondary task costs. Journal of Experimental Psychology: Learning, Memory, and Cognition, 31, 520-537.

Nettelbeck, T. (1987). Inspection time and intelligence. In P. A. Vernon (Ed.), Speed of information processing and intelligence (pp. 295-346). New York: Ablex.

Parkinson, S. R., Lindholm, J. M., \& Urell, T. (1980). Aging, dichotic memory, and digit span. Journal of Gerontology, 35, 87-95.

Pascual-Leone, J. (1970). A mathematical model for the transition rule in Piaget's developmental stages. Acta Psychologica, 32, 301-345.

Pelli, D. G. (1997). The VideoToolbox software for visual psychophysics: transforming numbers into movies. Spatial Vision, 10, $437-442$

Piek, J. P., Dyck, M. J., Nieman, A., Anderson, M., Hay, D., Smith, L. M., et al. (2004). The relationship between motor coordination, executive functioning and attention in school aged children. Archives of Clinical Neuropsychology, 19, 1063-1076.

Salthouse, T. A., Fristoe, N. M., Lineweaver, T. T., \& Coon, V. E. (1995). Ageing of attention: does the ability to divide decline? Memory \& Cognition, 23, 59-71.

Salthouse, T. A., Rogan, J. D., \& Prill, K. A. (1984). Division of attention: Age differences on a visually presented memory task. Memory \& Cognition, 12, 613-620.

Scheuffgen, K., Happé, F., Anderson, M., \& Frith, U. (2000). High "intelligence", low "IQ"? Speed of processing and measured IQ in children with autism. Development and Psychopathology, 12, 83-90.

Sebastian, M. V., Menor, J., \& Elosua, M. R. (2006). Attentional dysfunction of the central executive in $\mathrm{AD}$ : evidence from dual task. Cortex, 42, 1015-1020.

Somberg, B. L., \& Salthouse, T. A. (1982). Divided attention abilities in young and old adults. Journal of Experimental Psychology: Human Perception and Performance, 8, 651-663.

Sullivan, R., Senior, G., \& Hennessy, M. (2000, October). Australian age-education and premorbid cognitive/intellectual estimates for the WAIS-III. Paper presented at the 6th Annual Conference of the APS College of Clinical Neuropsychologists, Hunter Valley, Australia.

Thatcher, R. W. (1991). Maturation of the human frontal lobes: Physiological evidence for staging. Developmental Neuropsychology, 7, 397-419.

Thompson-Schill, S. L., Ramscar, M., \& Chrysikou, E. G. (2009). Cognition without control: When a little frontal lobe goes a long way. Current Directions in Psychological Science, 18, 259-263.

Verhaeghen, P., Steitz, D. W., Sliwinski, M. J., \& Cerella, J. (2003). Aging and dual-task performance: A meta-analysis. Psychology and Aging, 18, 443-460.

Wechsler, D. (1991). Wechsler intelligence scale for children-WISC III (3rd ed.). New York: Psychological Corp.

Wechsler, D. (1997). Wechsler adult intelligence scale -WAIS III (3rd ed.). San Antonio, TX: Harcourt Assessment.

Wright, R. E. (1981). Aging, divided attention, and processing capacity. Journal of Gerontology, 36, 605-614. 